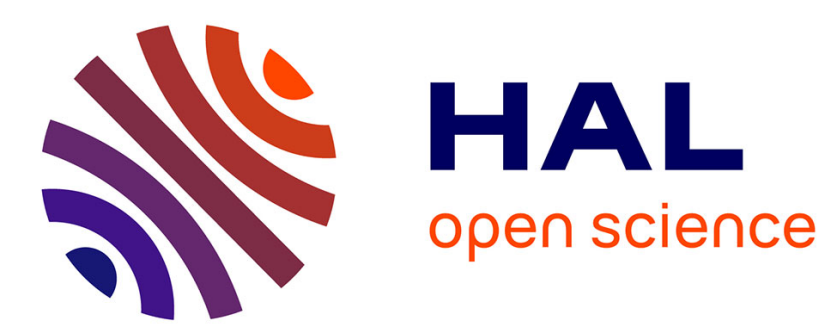

\title{
Coarse-grained simulations of charge, current and flow in heterogeneous media
}

Benjamin Rotenberg, Ignacio Pagonabarraga, Daan Frenkel

\section{To cite this version:}

Benjamin Rotenberg, Ignacio Pagonabarraga, Daan Frenkel. Coarse-grained simulations of charge, current and flow in heterogeneous media. Faraday Discussions, 2009, 144, pp.223. 10.1039/B901553A . hal-00531704

\section{HAL Id: hal-00531704 https://hal.science/hal-00531704}

Submitted on 9 Nov 2018

HAL is a multi-disciplinary open access archive for the deposit and dissemination of scientific research documents, whether they are published or not. The documents may come from teaching and research institutions in France or abroad, or from public or private research centers.
L'archive ouverte pluridisciplinaire HAL, est destinée au dépôt et à la diffusion de documents scientifiques de niveau recherche, publiés ou non, émanant des établissements d'enseignement et de recherche français ou étrangers, des laboratoires publics ou privés. 


\title{
Coarse-grained simulations of charge, current and flow in heterogeneous media
}

\author{
Benjamin Rotenberg ${ }^{1,2}$, Ignacio Pagonabarraga ${ }^{3}$, Daan Frenkel ${ }^{2,4}$ \\ 1 CNRS and UPMC-Paris6, Laboratoire PECSA, \\ UMR 7195, 4 place Jussieu, F-75005 Paris, France \\ 2 FOM Institute for Atomic and Molecular Physics, \\ Kruislaan 40\%, 1098 SJ Amsterdam, The Netherlands \\ 3 Departament de Fisica Fonamental, Universitat de Barcelona, \\ Carrer Martí i Franqués 1, 08028-Barcelona, Spain \\ 4 Department of Chemistry, University of Cambridge, \\ Lensfield Road, Cambridge CB2 1EW, United Kingdom
}

\begin{abstract}
We present a coarse-grained simulation method for complex charged systems. This mesoscopic model couples a hydrodynamic description to a free energy functional accounting for the interactions between solvent(s) and charged solutes. It is implemented in a hybrid lattice-based algorithm, whereby the evolution of the overall mass and momentum is taken care of via a Lattice Boltzmann scheme, whereas the composition and ionic concentrations are updated using the link-flux method. Several applications illustrate the power of this coarse-grained model for charged heterogeneous media : The transport of charged tracers in charged porous media, the deformation of an oil droplet in water under the effect of an applied electric field, and the distribution of ions at an oil-water interface as a function of their affinity for both solvents.
\end{abstract}

PACS numbers:

Keywords:

\section{INTRODUCTION}

The study of complex fluids and heterogeneous materials offers significant challenges because of the wide range of relevant length and time scales involved. Flow in porous media, the equilibrium and kinetic properties of membranes, oil/water mixtures or the electrokinetics of drops and colloids are examples of situations where the system evolves on scales much larger than molecular ones. Even the equilibrium structures characteristic of these systems are orders of magnitude larger than the molecular scale. Thus a simple atomistic description is not suitable and the use of soft coarse-grained potentials has become a standard alternative [1]. Under the action of external forces, the situation in these heterogeneous materials is even more involved, because of the hydrodynamic coupling between solutes and solvent. Hence, also the dynamic behaviour needs to be accounted for within a consistent coarse-grained approach.

A variety of strategies have been proposed to face these challenges. The combination of soft potentials and local thermostats which conserve momentum locally, as Dissipative Particle Dynamics [2] or the LoweAndersen thermostat [3], has allowed to reach hydrodynamic scales while keeping track of some microscopic details. Ideas from kinetic theory have also lead to flexible tools. Stochastic Rotation Dynamics couples molecular solutes to a coarse-grained solvent which recovers hydrodynamic behaviour [4]. Lattice Boltzmann (LB) [5], a method evolved from lat- tice gases to describe the hydrodynamics of fluids at long scales, has been extended to account for complex fluids. In particular, the combination of free energy based models coupled to a hydrodynamic description was introduced by Yeomans et al. [6-8] for simulations of non-ideal fluids and binary mixtures and later extended for other systems such as binary mixtures with surfactants [9-11] liquid crystals [12-15], ternary mixtures [16] or active fluids [17]. LB methods are particularly well suited for hydrodynamic simulations of fluids, especially in complex media, for it is in principle easy to parallelize the codes and to implement boundary conditions at solid/fluid interfaces [18]. This allowed the simulation of binary fluids in porous media [19], colloids at an oil-water interface [20-22] and suspensions of charged colloids [23].

Standard LB schemes for complex fluids often lead to spurious fluxes across solid-fluid boundaries or at liquid-liquid interfaces [24]. This can become catastrophic when one deals with charged solutes, as such fluxes could result in a progressive breakdown of electroneutrality. To overcome this difficulty, Capuani et al. $[25,26]$ introduced the link-flux method to reproduce the convective-diffusive dynamics of charged species in an electrolyte. Within this hybrid scheme, the overall mass and momentum of the fluid are evolved using a LB algorithm, whereas the ionic concentrations are updated using the link-flux method (for diffusion and migration) in combination with an advection scheme (for convection). This algorithm satisfies detailed balance at steady-state and allows to rigorously cancel fluxes into the solid, even in pres- 
ence of moving boundaries.

We want to put forward a general scheme to deal with complex charged fluids making use of this hybrid strategy, by combining the LB treatment of the solvent with a continuum treatment of binary charged fluids. We show how starting from a general free energy functional it is possible to obtain a dynamic scheme consistent with the prescribed thermodynamics and how it can be coupled to the LB method for the fluid flow. Several applications illustrate the power of this coarse-grained model for charged heterogeneous media : The transport of charged tracers in charged porous media, the deformation of an oil droplet in water under the effect of an applied electric field and the distribution of ions at an oil-water interface as a function of their affinity for both solvents. We compare with analytic predictions in the appropriate limits to illustrate that it is possible to achieve quantitative control of the performance of the model.

\section{FREE-ENERGY BASED MODEL OF NON-IDEAL MIXTURES}

In this section, we present the description of a complex mixture of solvents and charged solutes in terms of mesoscopic variables, the associated thermodynamic properties and the hydrodynamic equations governing their dynamics.

\section{Mesoscopic description}

Our aim is to describe charged solutes in heterogeneous media involving solid-liquid or liquid-liquid (e.g. oil-water) interfaces. For not too concentrated solutions, we can treat the fluid as a continuum, whose state is characterized by its local mass density $\rho(\mathbf{r}, t)$ and concentrations in each solute $\rho_{k}(\mathbf{r}, t)$. In the following we will always consider 1:1 electrolytes and denote the corresponding solutes by + and - for cations and anions, respectively. For oil-water mixtures, we also introduce the local composition

$$
\phi(\mathbf{r})=\frac{\rho_{o}(\mathbf{r})-\rho_{w}(\mathbf{r})}{\rho_{o}(\mathbf{r})+\rho_{w}(\mathbf{r})} \in[-1 ; 1]
$$

where the $w$ and $o$ subscripts refer to water and oil, respectively. Immiscible fluids are characterized by regions where $\phi$ is almost constant ( $\phi \sim-1$ in water and $\phi \sim+1$ in oil) separated by a "sharp" interface. The underlying assumption in the above description is to consider the fluid can be seen as locally homogeneous, although density, composition and solute concentrations can vary on a larger scale.

\section{Thermodynamics}

The thermodynamics of the system is determined by its free energy, expressed as the functional :

$$
\mathcal{F}\left[\phi, \rho_{+}, \rho_{-}\right]=\int \mathrm{d} \mathbf{r} \mathcal{F}_{V}\left[\phi(\mathbf{r}), \rho_{+}(\mathbf{r}), \rho_{-}(\mathbf{r})\right]
$$

where $\mathcal{F}_{V}$ is a free energy density. Following Onuki [27-29] we separate the contributions $\mathcal{F}^{\text {mix }}[\phi]$ describing the immiscible solvents and $\mathcal{F}^{\text {ions }}\left[\phi, \rho_{+}, \rho_{-}\right]$describing ions in a solvent of composition $\phi$. The mixing contribution is chosen of the Landau-Ginzburg form :

$$
\mathcal{F}^{\text {mix }}=\int \mathrm{d} \mathbf{r}\left[-\frac{1}{2} B \phi^{2}+\frac{1}{4} B \phi^{4}+\frac{1}{2} K(\nabla \phi)^{2}\right] .
$$

The first two terms correspond to the bulk phase behaviour, with minima for $\phi= \pm 1$, while the last reflects the cost of sustaining interfaces. The dimension of $B$ is energy $\times$ length $^{-3}$ and that of $K$ is energy $\times$ length $^{-1}$. This standard choice gives at equilibrium a planar interface of the form $\phi(x)=$ $\tanh (x / \xi)$ with a width $\xi=\sqrt{2 K / B}$ and a surface tension $\sigma=\sqrt{8 K B / 9}$.

The ionic contribution of the free energy consists of an ideal, a solvation and an electrostatic term :

$$
\begin{aligned}
& \mathcal{F}^{\text {ions }}=\int \mathrm{d} \mathbf{r} \sum_{\alpha= \pm} \rho_{\alpha}(\mathbf{r})\left[k_{B} T\left(\ln \rho_{\alpha}(\mathbf{r})-1\right)-\mu_{\alpha}\right. \\
& \left.+V_{\alpha}^{\text {solv }}(\mathbf{r})+\frac{z_{\alpha} e}{2} \psi(\mathbf{r})\right]
\end{aligned}
$$

where $z_{ \pm}= \pm 1$ is the valency of the ions, $\mu_{\alpha}$ is a reference chemical potential and the electrostatic potential $\psi$ is solution of the Poisson equation :

$$
\nabla \cdot[\epsilon(\mathbf{r}) \nabla \psi(\mathbf{r})]=-\left[\rho_{+}(\mathbf{r})-\rho_{-}(\mathbf{r})\right] e .
$$

The dielectric constant $\epsilon(\mathbf{r})$ depends on the local composition of the fluid. Although it could be a priori an intricate function of $\phi$, it is reasonable to assume a linear relation $\epsilon(\mathbf{r})=\bar{\epsilon}[1-\gamma \phi(\mathbf{r})]$ with $\bar{\epsilon}=\frac{\epsilon_{w}+\epsilon_{o}}{2}$ the average dielectric constant and $\gamma=\frac{\epsilon_{w}-\epsilon_{o}}{\epsilon_{w}+\epsilon_{o}} \in[0 ; 1]$ the dielectric contrast.

The ionic solvation potential $V_{ \pm}^{\text {solv }}$ accounts for the different solvation free energy in the two solvents. It is therefore natural to parametrize it as a function of the composition as $V_{ \pm}^{\text {solv }}(\mathbf{r})=\Delta \mu_{ \pm} \frac{1+\phi(\mathbf{r})}{2}$, where we have introduced for each ion the solvation free energy difference between water and oil $\Delta \mu_{ \pm}=\mu_{ \pm}^{o}-\mu_{ \pm}^{w}$, also referred to as extraction or Gibbs transfer free energy in the electrochemistry community. As the free energy (2) does not exclude in principle values of the composition parameter outside the $\phi \in[-1,1]$ 
range, we have considered that the physical properties $\left(\epsilon, V_{ \pm}^{\text {solv }}\right)$ for $\phi<-1$ are that of water $\left(\epsilon_{w}, 0\right)$ and for $\phi>1$ that of oil $\left(\epsilon_{o}, \Delta \mu_{ \pm}\right)$.

Let us now briefly analyze the properties of this free energy in terms of the chemical potentials associated to $\rho_{ \pm}$and $\phi$ and the corresponding thermodynamic forces acting on the fluid. The ionic chemical potentials are of the usual form :

$$
\mu_{ \pm}=\frac{\delta \mathcal{F}}{\delta \rho_{ \pm}}=k_{B} T \ln \rho_{ \pm}+V_{ \pm}^{s o l v}+z_{ \pm} e \psi
$$

while the chemical potential corresponding to the solvent mixture reads :

$$
\mu_{\phi}=\frac{\delta \mathcal{F}}{\delta \phi}=\mu_{\phi}^{m i x}+\mu_{\phi}^{s o l v}+\mu_{\phi}^{e l} .
$$

The first term is simply :

$$
\mu_{\phi}^{m i x}=-B \phi(\mathbf{r})+B \phi(\mathbf{r})^{3}-K \nabla^{2} \phi(\mathbf{r})
$$

When the solvation free energy is taken as $V_{ \pm}^{\text {solv }}(\mathbf{r})=$ $\Delta \mu_{ \pm} \frac{1+\phi(\mathbf{r})}{2}$ the second term is :

$$
\mu_{\phi}^{\text {solv }}=\frac{\left[\rho_{+}(\mathbf{r}) \Delta \mu_{+}+\rho_{-}(\mathbf{r}) \Delta \mu_{-}\right]}{2} .
$$

Finally, the electrostatic contribution is :

$$
\mu_{\phi}^{e l}=\frac{\mathbf{E}(\mathbf{r})^{2}}{2} \frac{\delta \epsilon}{\delta \phi}=-\frac{\gamma \bar{\epsilon}}{2} \mathbf{E}(\mathbf{r})^{2} .
$$

Excess chemical potential gradients give rise to a thermodynamic force (per unit volume) that can be expressed as a pressure gradient from the GibbsDuhem equality :

$$
\mathbf{f}_{V}^{t h}=-\nabla P=\phi \nabla \mu_{\phi}+\rho_{+} \nabla \mu_{+}^{e x}+\rho_{-} \nabla \mu_{-}^{e x}
$$

where the ex superscript refers to the excess chemical potentials. Each part of the chemical potentials (mixing, solvation and electrostatic) contribute to this force. For example, gradients of $\mu_{ \pm}^{e x}$ give rise to the force $\rho_{e l} \mathbf{E}$, with $\mathbf{E}=-\nabla \psi$ the electric field, and gradients of $\mu_{\phi}^{e l}$ are the source of the dielectrophoretic force $(\epsilon(\mathbf{r})-\bar{\epsilon}) \nabla\left(\frac{\mathbf{E}^{2}}{2}\right)$. The latter drives oil-rich fluid elements $(\phi>0, \epsilon<\bar{\epsilon})$ towards region where $\mathbf{E}^{2}$ is small and water-rich fluid elements towards region of higher $\mathbf{E}^{2}$ (note that $\nabla \mu_{\phi}^{e l}$ also generates a composition flux in addition to this force) and is particularly important in phenomena such as electrowetting. These electrostatic contributions to chemical potential gradients illustrate some new features captured by our free energy model compared to previous ones used in Lattice Boltzmann simulations of binary mixtures.

\section{Hydrodynamics}

The thermodynamic description of the system needs to be supplemented by a set of prescriptions for the dynamics. Overall mass conservation of the fluid implies :

$$
\partial_{t} \rho+\nabla \cdot(\rho \mathbf{u})=0
$$

with $\mathbf{u}$ the local barycentric velocity of the fluid. Momentum conservation of the fluid and viscous dissipation then enter in the Navier-Stokes equation, which reads for and incompressible fluid :

$$
\partial_{t} \mathbf{u}+\mathbf{u} \cdot \nabla \mathbf{u}=\nu \nabla^{2} \mathbf{u}+\frac{\mathbf{f}_{V}}{\rho}
$$

where $\nu=\eta / \rho$ is the kinematic viscosity and $\mathbf{f}_{V}=$ $\mathbf{f}_{V}^{e x t}+\mathbf{f}_{V}^{t h}$ is the sum of the externally applied force and thermodynamic force (11). Although we have not considered this in the following, it is in principle possible to introduce a composition-dependent viscosity [30].

The composition $\phi$ and ionic concentrations $\rho_{ \pm}$also satisfy conservation laws :

$$
\begin{aligned}
\partial_{t} \phi+\nabla \cdot(\phi \mathbf{u}) & =-\nabla \cdot \mathbf{j}_{\phi} \\
\partial_{t} \rho_{ \pm}+\nabla \cdot\left(\rho_{ \pm} \mathbf{u}\right) & =-\nabla \cdot \mathbf{j}_{ \pm}
\end{aligned}
$$

where we have introduced fluxes in the barycentric frame $\mathbf{j}_{\phi}$ and $\mathbf{j}_{ \pm}$. The latter are given by phenomenological equations, namely :

$$
\mathbf{j}_{ \pm}=-D_{ \pm} \rho_{ \pm} \nabla\left(\beta \mu_{ \pm}\right)
$$

with $D_{ \pm}$the ionic diffusion coefficients, and the CahnHilliard equation for the composition :

$$
\mathbf{j}_{\phi}=-M \nabla \mu_{\phi}
$$

with $M$ a mobility. The units of $M$ differ from those of $D_{ \pm}$and a diffusivity (in $\mathrm{m}^{2} \mathrm{~s}^{-1}$ ) of the interface can be defined as $D_{\phi}=M B\left(-1+3 \phi^{2}\right)$ with $B$ from (3). Note that near the interface $\phi \sim 0$ and $D_{\phi}<0$ : this "negative diffusion" maintains the composition jump at the interface.

\section{Discussion}

The above description of mixtures of solvent and ions and the particular choice of free energy functional are very similar to the ones adopted by Onuki [2729]. The functional differs only on two points. First, we follow previous LB studies of binary mixture $[18,20,31,32]$ and use the Landau-Ginzburg (LG) functional (3) instead of the Bragg-Williams one for numerical convenience. The combination of 
LG and electrostatic free energies (without solvation terms) has also been used to investigate the wetting of a solid substrate by ionic solutions [33]. The second difference with Onuki's approach consists in using $\rho_{e l} \psi$ for the electrostatic energy instead of $\epsilon(\nabla \psi)^{2}$. This more natural choice, consistent e.g. with the DFT work of van Roij et al. [34, 35], doesn't require any approximations for the treatment of "image charges".

A major difference with both Onuki's and van Roij's work is that the present model captures not only the equilibrium states of the system, but also its dynamics. It shares many features with Dynamical Density Functional Theory [36-39], since it relies on an expression of fluxes proportional to gradients of chemical potentials. The free energy functional described above is relatively simple, for it neglects e.g. the effect of the finite size of the ions and correlations beyond the mean-field level. This description is perfectly valid for dilute solutions of 1:1 electrolytes and can be improved if more concentrated solutions or multivalent ions are considered. Moreover, it captures the presence of immiscible solvents and the (possibly asymmetric) affinity of the ions for one of them. The free energy model put forward in this paper can be seen as a simple limiting case of more elaborate free energy functionals. The essential difference with DDFT is that the hydrodynamic behaviour of the fluid is properly described (it satisfies the Navier-Stokes equation resulting from momentum conservation and viscous dissipation), whereas most DDFT studies consider a fluid at rest or mediating hydrodynamic interactions between large solutes via effective interactions (Oseen or Rotne-Prager tensors) [40]. This latter approach can be efficient for suspensions of solid particles, but is not valid a priori for liquid droplets in another liquid.

\section{LATTICE SIMULATIONS}

The coarse-grained model introduced in the previous section couples a hydrodynamic description of the fluid to a free energy based representation of its thermodynamic behaviour. The purpose of the present section is to introduce the computational methods used to solve numerically the coupled evolution equations for the composition, ionic concentrations and fluid velocity. The general strategy relies on the use of different lattice models, which are described here successively.

\section{Hybrid lattice scheme}

For non-ideal multicomponent fluids, the standard LB approach treats all species on the same footing and populations (see below) are associated to each component. This can become computationally expensive for more than two components. Nevertheless, this approach has recently been applied for a mixture of two solvents and two reactive solutes by Furtado et al. [41] to study the convective drop motion driven by non-linear kinetics. An alternative is to use a hybrid LB / finite elements approach, whereby the LB fluid is described by populations evolving as before, but the order parameters (e.g. the composition $\phi$ ) are described by scalar fields evolving according to finite elements schemes [42, 43]. As mentioned in the introduction, such methods may suffer from spurious fluxes which motivated the development of the linkflux method by Capuani et al. [25, 26]. Here we generalize the link-flux approach to the more complex case of ions in a mixture of solvents. The overall mass and momentum are taken care of via a Lattice Boltzmann scheme, whereas the composition $\phi$ and ionic concentrations $\rho_{ \pm}$are updated using the link-flux method. We now develop these two steps.

\section{Lattice Boltzmann}

Lattice Boltzmann (LB) is a well established method for hydrodynamic simulations based on kinetic theory [5, 44]. The Boltzmann equation is a mesoscopic kinetic equation which determines the evolution of the probability density function $f(\mathbf{r}, \mathbf{v}, t)$ of finding a fluid particle with a velocity $\mathbf{v}$ at position $\mathbf{r}$ and time $t$. The hydrodynamic fluid variables are derived as moments in velocity space of the distribution function starting with the fluid local density $\rho=\int f \mathrm{~d} \mathbf{v}$ and mass flux $\rho \mathbf{u}=\int f \mathbf{v} \mathrm{d} \mathbf{v}$. Although the relaxation of the distribution function toward equilibrium is determined by a nonlinear collision operator, the Bhatnagar-Gross-Krook (BGK) model [45] shows that proper hydrodynamics can be already recovered from a linearized collision operator if it is isotropic and conserves mass and momentum.

LB tracks the dynamics of fluid populations, $f_{i}=$ $f\left(\mathbf{r}, \mathbf{c}_{\mathbf{i}}, t\right)$, which evolve on the nodes $\mathbf{r}$ of a lattice of spacing $\Delta x$ moving to neighbouring nodes at finite time steps through a finite set of allowed velocities $\left\{\mathbf{c}_{\mathbf{i}}\right\}_{i \in\left[1, N_{\max }\right]}$. The hydrodynamic variables are obtained as appropriate quadratures $\rho=\sum_{i} w_{i} f_{i}$ and $\rho \mathbf{u}=\sum_{i} w_{i} f_{i} \mathbf{c}_{\mathbf{i}}$, where the weights $w_{i}$ are associated to the chosen set of velocities. The particle distributions $f_{i}$ also relax at each time step toward a 
prescribed equilibrium distribution through a linear collision operator which conserves mass and momentum and ensures that the the solvent mass density $\rho$ and velocity $\mathbf{u}$ follow the Navier-Stokes equations, (12) and (13), on distances larger than $\Delta x$. The natural units in LB simulations are the lattice spacing $\Delta x$ and the time step $\Delta t$. They are fixed by the properties of the system: In the following, the lattice spacing is chosen as a fraction of the Bjerrum length $l_{B}=e^{2} / 4 \pi \epsilon k_{B} T$ (approximately $0.7 \mathrm{~nm}$ in water at room temperature), while the time step is determined by the value of the solvent viscosity.

The force per unit volume $\mathbf{f}_{V}$ acting on the fluid also enters in the collision rule. The issue of computing the thermodynamic force (11) from the state $\left(\phi, \rho_{ \pm}\right)$ of the system will be addressed in the next section. For numerical accuracy and stability reasons, the fluid velocity must remain small compared to the sound velocity $c_{s}$. This small Mach number limit implies that the forces are also small, i.e. that $\chi_{T} \mathbf{f}_{V} \Delta x \ll 1$, with $\chi_{T}$ the fluid compressibility $\left(\chi_{T}=1 / \rho c_{s}^{2}\right.$ for the LB fluid). Each term in the excess free energy density contributes to the force (11) and we can derive conditions accordingly. This leads for the $\mathcal{F}^{m i x}$ contribution (3) to $\chi_{T} B \ll \xi / \Delta x$ and similar requirements are obtained for the solvation and electrostatic ones.

\section{Link-flux method}

The composition of the fluid is characterized by the order parameters $\phi$ and $\rho_{ \pm}$and evolves according to Eqs. (14), (15) and (16). The numerical solution of these equations is achived by the link-flux method. This method was introduced by Capuani et al. [25, 26] in order to prevent spurious solute fluxes across solidfluid interfaces. It focusses on solute fluxes between lattice nodes rather than the amount of solute at each nodes. Integrating the conservation laws (14) over a volume corresponding to one lattice node and using Green's formula, we associate the variation of $\phi$ and $\rho_{ \pm}$to the fluxes of $\mathbf{j}_{\phi}$ and $\mathbf{j}_{ \pm}$across the surface $A_{0}$ of the cell (for more details, see Ref. [25]). The latter can be separated into the contributions of each link between the considered node and all its neighbours :

$$
\frac{\rho_{ \pm}(\mathbf{r}, t+\Delta t)-\rho_{ \pm}(\mathbf{r}, t)}{\Delta t} \Delta x^{3}=-A_{0} \sum_{i} j_{ \pm}^{i}(\mathbf{r})
$$

where $i$ refers to the discrete velocities and $j_{ \pm}^{i}$ to the contribution of link $i$ between $\mathbf{r}$ and $\mathbf{r}+\mathbf{c}_{\mathbf{i}} \Delta t$ to the outward flux of $\mathbf{j}_{ \pm}$. A similar expression can be written for the composition with link-fluxes $j_{\phi}^{i}$. In order to ensure that the ions follow a Boltzmann distribution at equilibrium, we rewrite (15) as $\mathbf{j}_{ \pm}=-D_{ \pm} e^{-\beta \mu_{ \pm}^{e x}} \nabla\left[\rho_{ \pm} e^{\beta \mu_{ \pm}^{e x}}\right]$ and express the linkfluxes in the symmetrized form :

$$
\begin{aligned}
& j_{ \pm}^{i}(\mathbf{r})=-d_{ \pm} \frac{e^{-\beta \mu_{ \pm}^{e x}(\mathbf{r})}+e^{-\beta \mu_{ \pm}^{e x}\left(\mathbf{r}+\mathbf{c}_{\mathbf{i}} \Delta t\right)}}{2} \\
& \quad \times\left[\frac{\rho_{ \pm}\left(\mathbf{r}+\mathbf{c}_{\mathbf{i}} \Delta t\right) e^{\beta \mu_{ \pm}^{e x}\left(\mathbf{r}+\mathbf{c}_{\mathbf{i}} \Delta t\right)}-\rho_{ \pm}(\mathbf{r}) e^{\beta \mu_{ \pm}^{e x}(\mathbf{r})}}{\Delta_{i}}\right]
\end{aligned}
$$

with $d_{ \pm}=D_{ \pm} / A_{0}$ and $\Delta_{i}=\left\|\mathbf{c}_{\mathbf{i}} \Delta t\right\|$. For links crossing solid-fluid boundaries (i.e. such that $\mathbf{r}+\mathbf{c}_{\mathbf{i}} \Delta t$ is a solid node) we enforce $j_{ \pm}^{i}=0$ so that such links do not carry any solute into the solid. For the solvent order parameter $\phi$ we use the simpler form :

$$
j_{\phi}^{i}(\mathbf{r})=-m_{\phi} \frac{\mu_{\phi}\left(\mathbf{r}+\mathbf{c}_{\mathbf{i}} \Delta t\right)-\mu_{\phi}(\mathbf{r})}{\Delta_{i}}
$$

with $m_{\phi}=M / A_{0}$ to recover the Cahn-Hilliard expression (16).

The link-flux algorithm just described takes care of the diffusive fluxes $\mathbf{j}_{\phi}$ and $\mathbf{j}_{ \pm}$. The advective fluxes $\phi \mathbf{u}$ and $\rho_{ \pm} \mathbf{u}$ are treated in a separate step described in detail in ref. [25]: It consists in transferring particles according to the overlap between the considered cell (around a node) translated by $\mathbf{u} \Delta t$ and each of its neighbouring cells. Finally, the update of the composition also modifies the local force (11) acting on the fluid. The thermodynamic force acting on node $\mathbf{r}$ is the average of the forces on each link $\mathbf{f}_{V}(\mathbf{r})=\sum_{i} w_{i} F_{i}(\mathbf{r}) \mathbf{c}_{\mathbf{i}}$, with :

$$
\begin{aligned}
F_{i}(\mathbf{r}) & =-\phi(\mathbf{r}) \frac{j_{\phi}^{i}(\mathbf{r})}{m_{\phi}} \\
& -k_{B} T \sum_{\alpha= \pm}\left[\frac{j_{\alpha}^{i}(\mathbf{r})}{d_{\alpha}}-\frac{\rho_{\alpha}\left(\mathbf{r}+\mathbf{c}_{\mathbf{i}} \Delta t\right)-\rho_{\alpha}(\mathbf{r})}{\Delta_{i}}\right]
\end{aligned}
$$

For the ionic part we substract the ideal term $\rho_{ \pm} \nabla \mu \pm^{i d}=\nabla \rho_{ \pm}$from the link flux, because only the excess (solvation and electrostatic) term $\rho_{ \pm} \nabla \mu_{ \pm}^{e x}$ contributes to the force.

The computation of the chemical potentials $\mu_{ \pm}$and $\mu_{\phi}$ require the evaluation at the lattice nodes of the electrostatic potential $\psi$ and its gradient $\mathbf{E}=-\nabla \psi$, as well as $\nabla^{2} \phi$ (see Eqs. (8) and (10)). The electrostatic potential is determined from the local charge and dielectric constant by solving the Poisson equation (5) with the Successive Over Relaxation (SOR) method [46], which also requires to compute gradients and Laplacian of $\phi$ and $\psi$. We have implemented a modified SOR algorithm with the additional term arising from the spatial variations of the permittivity. The following stencils are used to compute these 
differential operators :

$$
\begin{aligned}
\nabla \psi(\mathbf{r}) & =\frac{1}{\Delta t} \sum_{i} \frac{w_{i}}{c_{s}^{2}}\left[\psi\left(\mathbf{r}+\mathbf{c}_{\mathbf{i}} \Delta t\right)-\psi(\mathbf{r})\right] \mathbf{c}_{\mathbf{i}} \\
\nabla^{2} \psi(\mathbf{r}) & =\frac{2}{\Delta t^{2}} \sum_{i} \frac{w_{i}}{c_{s}^{2}}\left[\psi\left(\mathbf{r}+\mathbf{c}_{\mathbf{i}} \Delta t\right)-\psi(\mathbf{r})\right]
\end{aligned}
$$

This choice was motivated by numerical accuracy considerations, but also by the fact that it is fully consistent with the discretization used for the LB part of the hybrid scheme.

\section{CHARGED TRACERS IN CHARGED POROUS MEDIA}

We now show a first application of the method introduced in the previous section in which no oil is present. To assess the behaviour of a forced, charged fluid, we consider a slit of width $L$ with charged walls (charge density $\sigma^{e l}<0$ ) and compensating counterions in the fluid as a simple representation of a porous medium. In equilibrium, the counterion concentration is $\rho_{+}(x)=\frac{\alpha^{2}}{2 \pi l_{B}} \frac{1}{\cos ^{2} \alpha x}$, where $\alpha$ satisfies $\frac{\alpha L}{2} \tan \frac{\alpha L}{2}=\pi \sigma^{e l} L l_{B} / e$. If an electric field of strength $E_{0}$ is applied parallel to the solid walls, an electroosmotic flow develops in the confined fluid,

$$
u_{y}(x)=u_{r e f} \ln \frac{\cos \alpha x}{\cos \alpha L / 2}
$$

where the amplitude satisfies $u_{r e f} \equiv e E_{0} / 2 \pi \eta l_{B}$. In Fig. 1 we compare the theoretical predictions and the computed values for the flow field for increasing charge of the solid walls. One can see that the method describes quantitatively the osmotic flow deep into the non-linear regime of electrostatic coupling $(\alpha L \rightarrow \pi)$. Previous work has shown that other dynamic quantities, such as the dispersion of charged tracers by this flow, can be easily recovered [47].

A very useful quantity to analyze the diffusive dynamics in porous media is the time-dependent diffusion coefficient $D(t)$, that can be measured by NMR [48]. At short times $D(t)$ coincides with the molecular diffusion coefficient $D$. For neutral tracers, the short-time behaviour of $D(t)$ reflects the geometry of the pores [49]: $\frac{D(t)}{D} \sim 1-\frac{4}{9 \sqrt{\pi}} \frac{S}{V_{p}} \sqrt{D t}$ with $V_{p}$ the pore volume, $S$ the surface of the solid. The long time limit of $D(t)$ is the effective diffusion coefficient :

$$
D_{e}=\lim _{t \rightarrow \infty} D(t)
$$

which reflects the connectivity between pores throughout the medium. The ratio $D_{e} / D$ is often referred to as the inverse of the tortuosity. The effective tracer

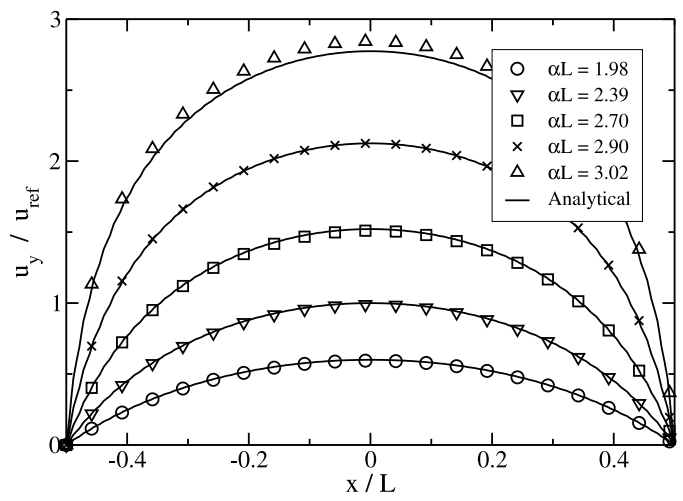

FIG. 1: Steady state flow profile across a slit of width $L=60 \Delta x$ (with $l_{B}=0.4 \Delta x$ ) for an applied electric field of magnitude $\beta e E_{0} L=3$ as a function of the the solid surface charge density. Symbols are simulation results while the continuous curves correspond to the theoretical prediction.

diffusion coefficient through charged porous materials is known to depend on its charge. For example, it has been observed in clays (negatively charged minerals) that the ratio $D_{e} / D$ for cations is larger than for neutral tracers, whereas that for anions is smaller [50-52]. This can be at least partly explained by the so-called Donnan effect : The concentration of co-ions (resp. counterions) in the pores of the material is smaller (resp. larger) than the concentration of salt in the reservoirs used to impose a concentration gradient to the sample, and this modifies the effective concentration gradient inside the sample.

Even if we correct for this effect, we expect that ions of different charge will follow different pathways through the pores and this might influence the observed value of $D_{e}$. In the framework of the proposed model, we can analyze $D(t)$ as the integral of the tracer's velocity autocorrelation function (VACF) using the Moment Propagation [47, 53-55]. It is worth emphasizing that the present analysis is possible because we are able to evaluate $D(t)$ numerically. Although this has been done for neutral tracers by averaging over trajectories of explicit tracer particles [56], we found no such analysis for charged tracers.

\section{System}

We will analyze the diffusion of charged tracers in a porous medium consisting of a compact FCC lattice of charged spheres of radius $R$ with a surface charge density $\sigma_{e l}<0$ whose pores are saturated with an electrolyte solution of concentration $\rho_{b}$. The void frac- 
tion (porosity) of $1-\pi / 3 \sqrt{2} \sim 26 \%$ is divided into large octahedral $\left(O_{h}\right)$ cavities of radius $r_{O_{h}} \sim 0.41 R$ connected by smaller tetrahedral $\left(T_{d}\right)$ pores of radius $r_{T_{d}} \sim 0.22 R$. The size of the bottlenecks between $O_{h}$ and $T_{d}$ pores is approximately $0.15 R$. The electrostatic potential distribution inside the pore is controlled by the salt concentration, with a typical double-layer thickness $\kappa_{b}^{-1}=\left(8 \pi l_{B} \rho_{b}\right)^{-1 / 2}$, with $l_{B}$ the Bjerrum length. The Debye length $\kappa_{b}^{-1}$ corresponds to the exponential decay of the potential near a planar interface, for not too high a surface potential $\psi_{S}$ (compared to $k_{B} T / e$ and using the potential of the solution "far" from the surface as a reference). The latter depends on the surface charge density and the salt concentration in the medium.

Simulations were performed on a $a^{3}=(100 \Delta x)^{3}$ lattice, with spheres of radius $R=a / 2 \sqrt{2} \sim 35.4 \Delta x$. The lattice spacing is $2.5 l_{B} \sim 1.75 \mathrm{~nm}$, so that the $O_{h}$ (resp. $T_{d}$ ) pore size is $\sim 25.8 \mathrm{~nm}$ (resp. $13.9 \mathrm{~nm}$ ). The charge density of the solid is $\sigma_{e l} \sim-0.04 \mathrm{e} / \mathrm{nm}^{2}$, and we considered salt concentrations $\rho_{b}$ corresponding to $\left(\kappa_{b} R\right)^{-1} \in[0.02 ; 0.57]$. The molecular diffusion coefficients are $D_{ \pm}=510^{-2}\left(\Delta x^{2} / \Delta t\right)$. The system is initialized with cations and anions distributed homogeneously and evolved until the equilibrium distribution is reached.

\section{Results and discussion}

The time-dependent diffusion coefficient $D(t)$ for tracers of valency \pm 1 and 0 with the same molecular diffusion coefficient $D$ are computed using the moment propagation method. The results show that the charge of the ion influences both the value of $D_{e}$ and the transient regime to reach this asymptotic value. $D_{e} / D$ is larger (resp. smaller) for cations (resp. anions) than for neutral tracers. This is in agreement with experimental observations (which also reflect the Donnan effect). $D(t)$ also tends towards $D_{e}$ faster (resp. more slowly) for cations (resp. anions) than neutral tracers. This can be quantified by a characteristic time :

$$
\tau=\int_{0}^{\infty} \frac{D(t)-D}{D_{e}-D} \mathrm{~d} t
$$

Fig. 2 shows the variation of tracer's diffusion and the relaxation time $\tau$ with salt concentration $\rho_{b}$. The extension of the diffuse layer (approximately $\kappa_{b}^{-1}$ ) decreases with increasing $\rho_{b}$. Both $D_{e}$ and $\tau$ for the charged tracers tend towards the values for neutral tracers at high $\rho_{b}$. This effect has been observed in diffusion experiments (also reflecting the smaller Donnan effect in that case). To our knowledge, there is no experimental measurements of time-dependent diffusion coefficient for ions in clays.

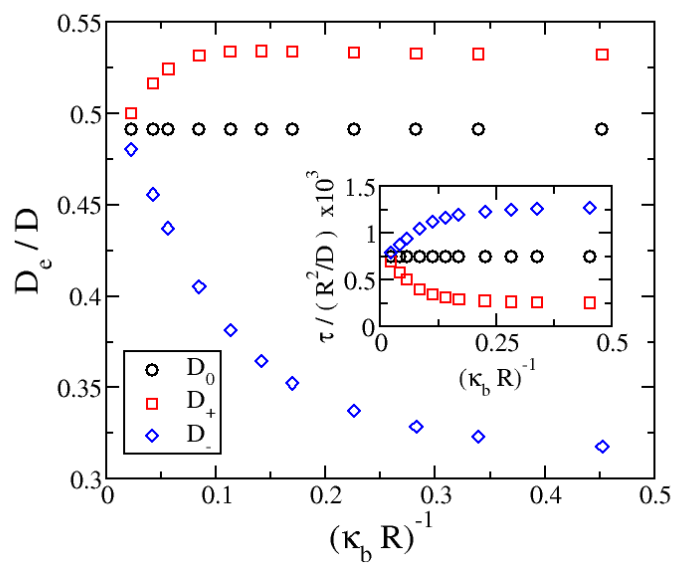

FIG. 2: Effective diffusion coefficient for charged tracers, as a function of the salt concentration. The results for neutral (॰), cationic $(\square)$ and anionic $(\diamond)$ tracers , normalized by the molecular diffusion coefficient $D$, are reported as a function of the equivalent Debye length in a bulk solution $\kappa_{b}^{-1}=\left(8 \pi l_{B} \rho_{b}\right)^{-1 / 2}$ divided by the radius $R$ of the spheres. The insert shows the characteristic time to explore the porosity accessible to each tracer, normalized by $R^{2} / D$.

The variations of $D_{e} / D$ and $\tau$ with the tracer charge and salt concentrations reflect how tracers go from one cavity to another to explore the whole porosity. Smaller $T_{d}$ pores act as bottlenecks through which tracers must pass to go from one $O_{h}$ pore to another. In addition to this purely geometric (entropic) effect, the electrostatic potential distribution in the porosity also affects the motion of charged tracers. The electrostatic potential $\psi(\mathbf{r})$ in the pore is always larger than the surface potential $\psi_{S}$ and increases with increasing distance from the surfaces. Thus $\psi$ is larger near the center of $O_{h}$ pores (see the disconnected isopotential surfaces for a large $\psi$, in red in Fig. 2) than in $T_{d}$ pores and anions feel a repulsive electrostatic force when approaching the latter. This decreases the probability to go from one $O_{h}$ cavity to the next and consequently $D_{e}$ is smaller than for netral tracers. This also implies that it takes longer for anions to explore the volume accessible to them (although this volume is smaller than for neutral species that are not repelled from the surfaces) and the corresponding $\tau$ is larger.

As opposed to anions, cations accumulate near the surface. As can be seen in Fig. 3, the diffuse layer forms a continuous volume throughout the porosity and cations can follow preferential pathways along the surfaces. This surface diffusion mechanism is more 


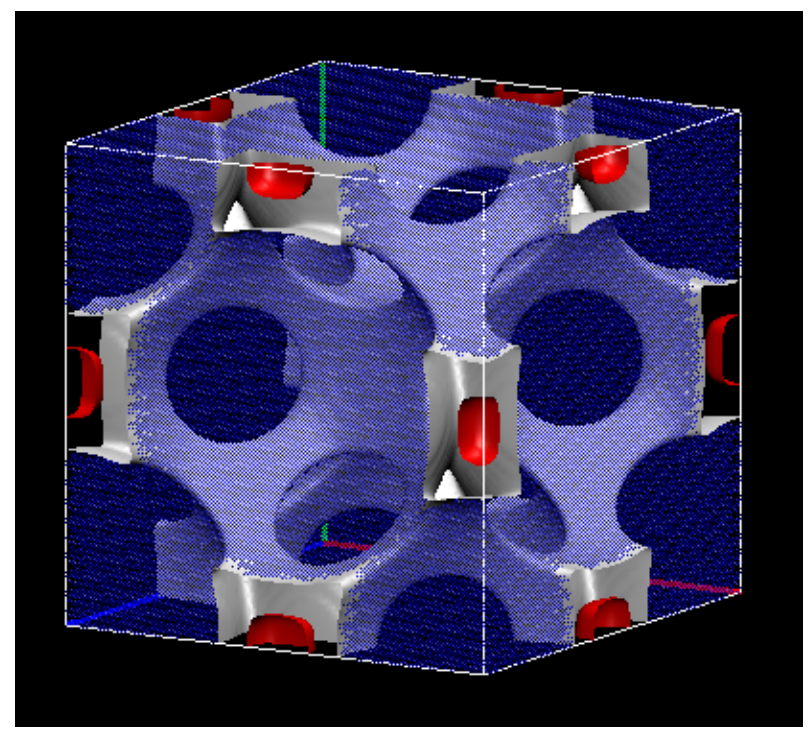

FIG. 3: Isopotential curves for a FCC lattice of charged spheres where the porosity contains solvent, counterions and salt. The grey surface is an electric isopotential curve for a value close to the surface potential while the red surfaces correspond to a larger value of the electrostatic potential. Each lattice node in the solid phase is represented as a blue dot, while fluid nodes are not indicated for clarity.

efficient than for neutral cations, since exploring a smaller volume takes less time than exploring the whole $O_{h}$ pores. Hence the larger $D_{e}$ and smaller $\tau$ for cations. In both cases, there is a clear interplay between the geometric and electrostatic effects. At higher concentrations, the potential variations are screened and the effect of the above-mentioned mechanisms are less pronounced.

\section{DIELECTRIC DROPLETS UNDER AN ELECTRIC FIELD}

In this section, we apply our mesoscopic model to the deformation of an oil droplet in water under an applied electric field, in the absence of ions. Because of surface tension, the equilibrium shape of an oil droplet in water corresponds to the minimal interface area (a disk in 2D, a sphere in 3D) and there exists an excess (Laplace) pressure inside the drop : $P_{L}=\frac{\sigma}{R_{d}}$ in $2 \mathrm{D}$ and $\frac{2 \sigma}{R_{d}}$ in $3 \mathrm{D}$, with $\sigma$ the surface tension and $R_{d}$ the drop radius. When the drop and the suspending liquid have different dielectric constants (i.e. when $\left.\gamma=\frac{\epsilon_{w}-\epsilon_{o}}{\epsilon_{w}+\epsilon_{o}} \neq 0\right)$, applying an electric field $E$ polarizes the drop and the anisotropic electrostatic stress tensor tends to elongate it in the direction of the field.
The final shape is governed by the balance between electrostatic and surface tension forces. For small applied fields the equilibrium shape is an ellipse in 2D (an ellipsoid in 3D) and the deformation is defined as $\mathcal{D}=(b-a) /(b+a)$ with $b$ (resp. $a)$ its large (resp. small) axis. In the small $E$ limit, an analytical result for the deformation in the $2 \mathrm{D}$ case can be obtained following the lines of [57] for spherical droplets, with the result (see below):

$$
\mathcal{D}_{\text {theor }}=\frac{1}{4} \gamma^{2}(1+\gamma) \frac{\bar{\epsilon} E^{2} R_{d}}{\sigma}
$$

where $\bar{\epsilon}=\left(\epsilon_{w}+\epsilon_{o}\right) / 2$.

\section{System}

We performed simulations of a two-dimensional oil droplet in water. Because of periodic boundary conditions in all directions, this corresponds to an array of infinite cylinders. The box size is $N \times N \times 1$ lattice points, with $N=50$ or 100 . The parameters entering in the free energy (3) are $\beta B \Delta x^{3}=10^{-3}$ and $\beta K \Delta x=310^{-3}$, giving a theoretical interface width of $\xi=\sqrt{2 K / B} \sim 2.45 \Delta x$. and a surface tension $\beta \sigma \Delta x^{2}=\sqrt{8 K B / 9} \sim 1.6310^{-3}$. This choice of parameters ensures that the interface is thin while remaining well resolved on the lattice. In particular, we checked that in the case of a planar interface (1D geometry) the simulated systems reproduces accurately the $\phi(x)=\tanh (x / \xi)$ profile with the expected width. These parameters also fulfill the condition $\chi_{T} B \ll \xi / \Delta x$. The interface mobility $M$ is such that $M B=10^{-2} \Delta x^{2} / \Delta t$.

After equilibrating the system, we turn on the electric field $E$ by solving the Poisson equation under the condition of a potential drop $-E L$ between both sides of the simulation box (to be consistent with the periodic boundary conditions). The electrostatic potential inside and outside the droplet is reported in Fig. 4 together with the correspongindg electric field lines, for a large dielectric contrast $\gamma=0.9$ and an applied electric field $\beta e E \Delta x=10^{-3}$. The average dielectric constant $\bar{\epsilon}=\left(\epsilon_{w}+\epsilon_{o}\right) / 2$ is such that $\bar{l}_{B}=\beta e^{2} / 4 \pi \bar{\epsilon}=0.4 \Delta x$. Simulation results for two system sizes are shown : The smaller (b) corresponds to a box size $L=50 \Delta x$ and a droplet radius $R_{d}=14.1 \Delta x$, the larger (d) to $L=100 \Delta x$, $R_{d}=28.2 \Delta x$. These results are compared to the analytical solution for an isolated cylinder :

$$
\begin{array}{ll}
\mathbf{E}(\mathbf{r})=(1+\gamma) \mathbf{E}_{0} & , \quad r<R_{d} \\
\mathbf{E}(\mathbf{r})=\mathbf{E}_{0}+\gamma R_{d}^{2} \nabla\left(\frac{\mathbf{E}_{0} \cdot \mathbf{r}}{r^{2}}\right) & , \quad r>R_{d}
\end{array}
$$




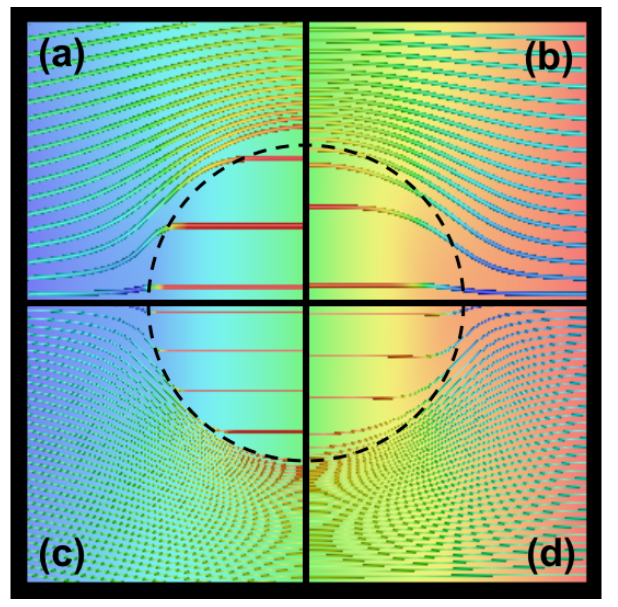

FIG. 4: Electrostatic potential (background) and electric field lines (the color of streamlines indicates the magnitude of the field, with red for large fields) for an oil droplet in water under an electric field. The oil-water interface is indicated as a dashed line. The dielectric constrast is $\gamma=0.9$. Analytical results for an isolet droplet (a and c) are compared to simulation results with periodic boundary conditions ( $\mathrm{b}$ and $\mathrm{d}$ ). The effect of the finite width $(\xi=$ $2.45 \Delta x$ for $\mathrm{b}$ and $\mathrm{d}$ ) is more visible for the smaller system (a,b: box size $L=50 \Delta x$, droplet radius $R_{d}=14.1 \Delta x$ ) than with the larger one (c,d: $\left.L=100 \Delta x, R_{d}=28.2 \Delta x\right)$.

with $\mathbf{E}_{0}$ the applied field, evaluated on the nodes of the same lattices ( $a$ and $c$ ). Each part of the figure represents $1 / 4$ of the simulation box. The figures for the smaller system ( $a$ and $b$ ) have been magnified to appear of the same size as the larger one (c and d). The field lines are computed numerically from the electrostatic potential and colored according to the magnitude of the local electric field.

The simulation results for a system with periodic boundary conditions are in good agreement with the analytical solution for an isolated droplet. In particular, the field lines tend to bypass the less dielectric droplet and are thus closer to each other in the water phase. The field strength is smaller in the water phase, where the dielectric constant is higher. The analytical solution (26) corresponds to an infinitely thin interface $(\xi=0)$ with a discontinuous dielectric constant, whereas in simulations the latter varies smoothly over a distance $\sim \xi$. The effect of the finite width is more pronounced for the smaller system $\left(\xi / R_{d} \sim 0.17\right)$ and the agreement with $(26)$ is excellent for the larger $\left(\xi / R_{d} \sim 0.09\right)$. Although smaller $\xi / R_{d}$ can be reached by decreasing $\xi$, the latter should remain at least of a few $\Delta x$ for the variations of the composition $\phi$ to be well resolved on the lattice.

\section{Results and discussion}

Under the applied electric field, gradients of $\mu_{\phi}$ lead to an evolution of the composition $\phi$, corresponding to a deformation of the droplet. The precise location of the interface is defined by the curve $\phi(\mathbf{r})=0$, which is fitted to an ellipse to obtain the deformation $\mathcal{D}$. Results are reported in Fig. 5 as a function of the dielectric contrast $\gamma$ for two droplet radii and two electric field strengths. For the simulation parameters used here, the largest deformation is $<10^{-2}$ so that we are always in the small deformation limit assumed in Eq. (25). Simulation results are in excellent agreement with the analytical ones. This demonstrate that the simulation method faithfully reproduces the prediction of the continuous free energy model.

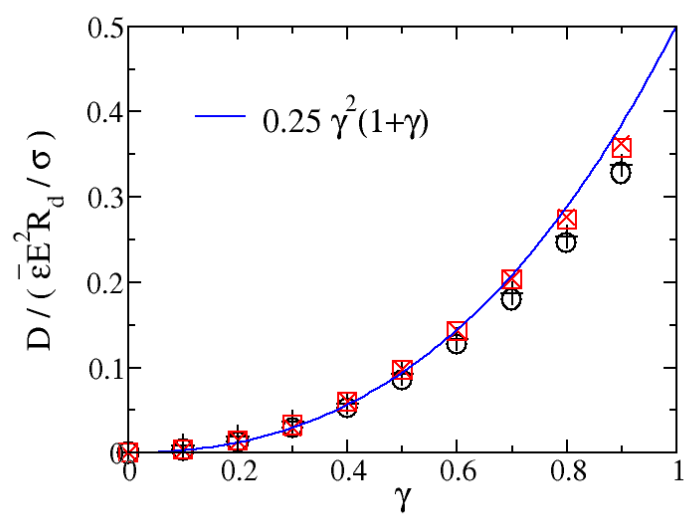

FIG. 5: Deformation of a 2D-oil droplet in water, as a function of the dielectric contrast $\gamma=\left(\epsilon_{w}-\epsilon_{o}\right) /\left(\epsilon_{w}+\epsilon_{o}\right)$. The deformation $\mathcal{D}$ is normalized by $\bar{\epsilon} E^{2} R_{d} / \sigma$, with $\bar{\epsilon}=$ $\left(\epsilon_{w}+\epsilon_{o}\right) / 2, E$ the applied field, $R_{d}$ the droplet radius and $\sigma$ the surface tension. Simulation results are for two droplet radii $\left(R_{d}=14.1 \Delta x\right.$ for $\square, \times$ and $28.2 \Delta x$ for $\circ$, $+)$ and two electric field strengths $\left(\beta e E \Delta x=10^{-3}\right.$ for $\circ, \square$ and $310^{-3}$ for $\times,+$ ) with the same interface width $\xi=2.45 \Delta x$. The solid line corresponds to the analytical solution (25).

The deviations observable only for large values of $\gamma$ depend mainly on the radius $R_{d}$ and less significantly on the applied electric field $\mathbf{E}$. This can be understood by analyzing the effect of the finite width on the energy balance leading to the final shape of the droplet and the theoretical deformation (25). In the case of a thin interface $(\xi=0)$ and assuming that the field at steady-state is still given by (26), we find that the electrostatic energy stored in the droplet when the field is turned on is (per unit length of the cylinder) : $\int_{\text {drop }} \frac{1}{2}\left(\epsilon_{o} E_{\text {in }}^{2}-\epsilon_{w} E_{0}^{2}\right) \mathrm{d} S=-\frac{1}{2} \bar{\epsilon}(1+\gamma) \gamma^{2} E_{0}^{2} S$, with 
$S=\pi R_{d}^{2}$ the section area of the droplet. This term tends to increase $S$ by deforming the droplet and is balanced by the increase in surface energy $\sigma 2 \pi R_{d} \mathcal{D}$ (per unit length of the cylinder). The corresponding deformation $\mathcal{D}$ is given by Eq. (25).

The main effect of the finite width $\xi$ is that the smooth variation of $\epsilon$ at the interface leads to a $\mathcal{O}\left(\xi R_{d} \bar{\epsilon}(1+\gamma) \gamma^{2} E_{0}^{2}\right)$ correction to the electrostatic term, resulting in a smaller driving force for the deformation. This explains the field-independent $\mathcal{O}\left(\xi / R_{d}\right)$ deviation of the simulation results from $\mathcal{D}_{\text {theor }} /\left(\bar{\epsilon} E^{2} R_{d} / \sigma\right)$ observed in Fig. 5. Other possible field-dependent corrections do not seem to be observed.

The results shown in this section demonstrate that the lattice simulation scheme is able to reproduce quantitatively the behaviour of a system consisting of two immiscible dielectric fluids under an electric field. We have not considered here the possibility of altering the phase behaviour of the solvent mixture in an inhomogeneous electric field, but this phenomenon is also captured by the free energy model used for our simulations [58].

\section{IONS AT AN OIL-WATER INTERFACE}

In this last section, we further exploit the power of our coarse-grained description by considering ions at a planar oil-water interface. The two solvents may have different dielectric constants and the ions different affinities for the two solvents $\left(\Delta \mu_{ \pm}=\mu_{ \pm}^{o}-\mu_{ \pm}^{w} \neq 0\right)$. The salt is partioned between the two phases and the salt concentration ratio is $\rho^{o} / \rho^{w}=\exp \left(-\beta \Delta \mu_{a v}\right)$ with $\Delta \mu_{a v}=\left(\Delta \mu_{+}+\Delta \mu_{-}\right) / 2$. In the case of asymmetric solvation $\left(\Delta \mu_{+} \neq \Delta \mu_{-}\right)$there exists at equilibrium an electrostatic potential difference (Donnan potential) across the interface $\psi_{D}=\psi^{o}-\psi^{w}=\left(\Delta \mu_{-}-\Delta \mu_{+}\right) / 2 e$. In that case there is a charge separation over distances characterized by two different Debye lengths $\kappa_{o, w}^{-1}=\left(2 \beta e^{2} \rho^{o, w} / \epsilon_{o, w}\right)^{-1 / 2}$. For large values of the dielectric contrast or of $\Delta \mu_{a v}$, they can differ by several orders of magnitude.

Experimental investigations of such interfaces at the microscopic scale have revealed that the interplay between solvation and electrostatic forces at the interface is rather complex [59]. This is particularly true if there is a dielectric constrast between the two phases $(\gamma \neq 0)$ resulting in image charges interactions. While Onuki accounted for them by an effective "image charge potential" in his free energy description of mixtures of oil, water and ions [27, 28], van Roij et al. [35] suggested a Poisson-Boltzmann (PB) treatment for a thin planar interface introducing a shift $s$ between the true solvent interface (where the dielectric constant changes) and the location of the jump $\Delta \mu_{ \pm}$in solvation potential felt by the ions. When $x<0$ (resp. $x>0$ ) corresponds to the oil (resp. water) phase, assuming $s>0$ and setting $\psi(\infty)=0$, we can write their result for the electrostatic potential as :

$$
\psi^{P B}=\left\{\begin{array}{cc}
\psi_{D}-\frac{\psi_{D}}{A} e^{\kappa_{o} x}, & x<0 \\
\psi_{D}-\frac{\psi_{D}}{A}\left[\cosh \left(\kappa_{i} x\right)+\right. & \left.n \sinh \left(\kappa_{i} x\right)\right], \\
& x \in[0, s] \\
\frac{\psi_{D}}{A} e^{-\kappa_{w}(x-s)} p\left[n \cosh \left(\kappa_{i} s\right)+\sinh \left(\kappa_{i} s\right)\right] & x>s
\end{array}\right.
$$

where $A=(1+n p) \cosh \left(\kappa_{i} s\right)+(n+p) \sinh \left(\kappa_{i} s\right)$, $n=\sqrt{\epsilon_{o} / \epsilon_{w}}, p=\sqrt{\rho^{o} / \rho^{w}}=\exp \left(-\beta \Delta \mu_{a v} / 2\right)$ and the screening length in the intermediate region is $\kappa_{i}^{-1}=\left(2 \beta e^{2} \rho^{o} / \epsilon_{w}\right)^{-1 / 2}$. This analytical result is exact only in the linearized regime, i.e. potential differences small compared to $k_{B} T / e$. Here we report lattice simulations based on our free energy model and compare the resulting ionic profiles to the prediction of the PB treatment for a flat interface.

\section{System}

We peformed simulations of flat oil-water interfaces in a simulation box consisting of $N \times 1 \times 1$ lattice points with $N=500$ and containing two interfaces (one $\mathrm{O} / \mathrm{W}$ and one $\mathrm{W} / \mathrm{O}$ ). Owing to the periodic boundary conditions, the system corresponds to an infinite stack of oil and water slabs of width $2 L_{o}$ and $2 L_{w}$ (we used $\left.L_{o}=L_{w}=125 \Delta x\right)$. There are several relevant lengthscales in the system: The interface width $\xi$, the Debye screening lengths $\kappa_{o, w}^{-1}$ and the size of both phases $L_{o, w}$. The width of the interface should be small compared to all other lengthscales : $\kappa_{o, w} \xi \ll 1$ and $\xi \ll L_{o, w}$. In principle, the electric double-layers can overlap, especially in the oil phase where $\kappa_{o}^{-1}$ is larger. This is even a crucial point to explain the electrostatic stabilization of surfactant free water droplets in oil $[60,61]$. In the present paper, we want to assess the validity of our coarse-grained simulation method by comparison with known results for an isolated interface and therefore consider only the limit $\kappa_{o, w} L_{o, w} \rightarrow \infty$. In particular, this condition is necessary for the salt concentrations to reach bulk values $\rho^{o, w}$ corresponding to well-defined screening lengths. Otherwise the amount of ions in the double layers could be non-negligible compared to the 
amount of ions in the bulk oil and water phases and the latter couldn't be considered as reservoirs. All simulations were performed using mixing free energy parameters $\beta B \Delta x^{3}=10^{-2}$ and $\beta K \Delta x=310^{-2}$ with a mobility such that $M B=510^{-2} \Delta x^{2} / \Delta t$. These values of $B$ and $K$ lead in the absence of ions to an interface width $\xi \sim 2.45 \Delta x$. Results are given only for one of the two interfaces in the simulation box, with $x<0$ (resp. $x>0$ ) corresponding to the oil (resp. water) phase.

\section{Results and discussion}

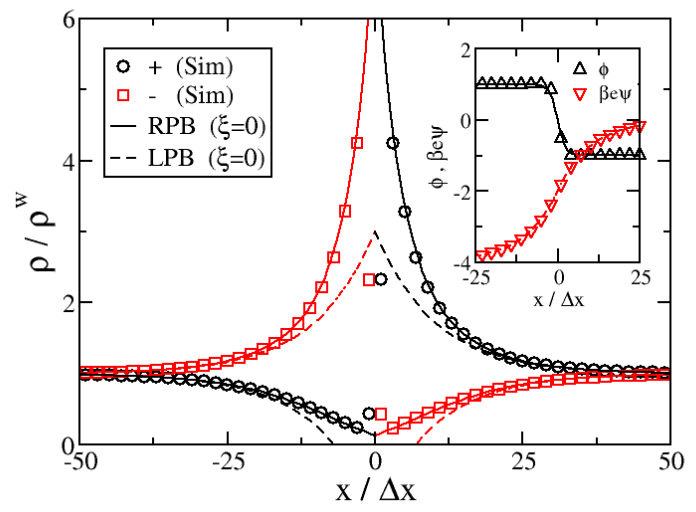

FIG. 6: Ionic profiles at an oil (left) - water (right) interface, for $\gamma=0, \beta \Delta \mu_{ \pm}= \pm 4, l_{B}=0.8 \Delta x$ and $\rho^{w} \Delta x^{3}=4.710^{-4}$. Symbols are simulation results. Lines are analytical results based on Eq. (27) with a shift $s=0$ for the electrostatic potential $\psi^{P B}$. Dashed lines corresponds to the fully lineared solution, solid lines to the reexponentiated one (see text). The insert compares the simulation results (symbols) for $\phi$ and $\psi$ to the analytical solutions (lines).

We first investigate the influence of the solvation free energy differences $\Delta \mu_{ \pm}$on the structure of the interface by considering a system without image charges $\left(\epsilon_{w}=\epsilon_{o}\right.$, i.e. $\left.\gamma=0\right)$. Fig. 6 compares simulation results to analytical predictions based on Eq. (27) for $\beta \Delta \mu_{ \pm}= \pm 4$, corresponding to hydrophilic cations and hydrophobic anions. From the PB ionic chemical potential $\mu_{ \pm}^{P B}= \pm e \psi^{P B}+V_{ \pm}^{\text {solv }}$ we compute the ionic concentrations either as $\rho_{ \pm}=\rho^{w} e^{-\beta \mu_{ \pm}^{P B}}$ or as the linear expansion $\rho^{w}\left(1-\beta \mu_{ \pm}^{P B}\right)$. In the following, we refer to the former as the reexponentiated $\mathrm{PB}$ result (RPB, see e.g. Ref. [62]) and to the latter as the linearized PB result (LPB). Since $\beta \Delta \mu_{a v}=0$ we expect equal salt concentrations in both phases and equal screening lengths (since we also have $\gamma=0$ ) $\kappa_{o, w}^{-1} \sim 10.3 \Delta x$, with a relatively good separation of length scales $\left(\kappa_{o, w} \xi<1\right.$ and $\left.\kappa_{o, w} L_{o, w}>1\right)$. We also expect a Donnan potential difference $\psi_{D}=-4 k_{B} T / e$ across the interface.

This figure shows that the LPB approximation fails to reproduce the ionic profiles, as expected for this large value of $\beta e \psi_{D}$. It even predicts negative concentrations near the interface. Adding the quadratic term in $\beta e \psi_{D}$ to approximate $\rho_{ \pm}$significantly improves the agreement while remaining consistent with the linear approximation for the potential (not shown). The RPB result, although not fully consistent with this approximation, is in quantitative agreement with the simulations, except at the interface where the finite width $\xi$ smoothens the jump in ionic concentrations. The insert of Fig. 6 shows that the composition profile is close to $\phi^{t h}(x)=-\tanh (x / \xi)$ and that the electrostatic potential is very well described by the linearized PB solution $\psi^{P B}(x)$ with a shift $s=0$. Note that Eq. (27) for $\psi^{P B}$ is obtained under the assumption $\xi=0$, and that potential differences are small compared to $k_{B} T / e$. Surprizingly, simulations for a finite $\xi$ with relatively large values of $\beta e \psi_{D}$ give similar results for the electrostatic potential even in the transition region where the ionic profiles differ. Although it is not easily seen on this insert, the simulated profile is in fact sharper than $\phi^{t h}(x)$.

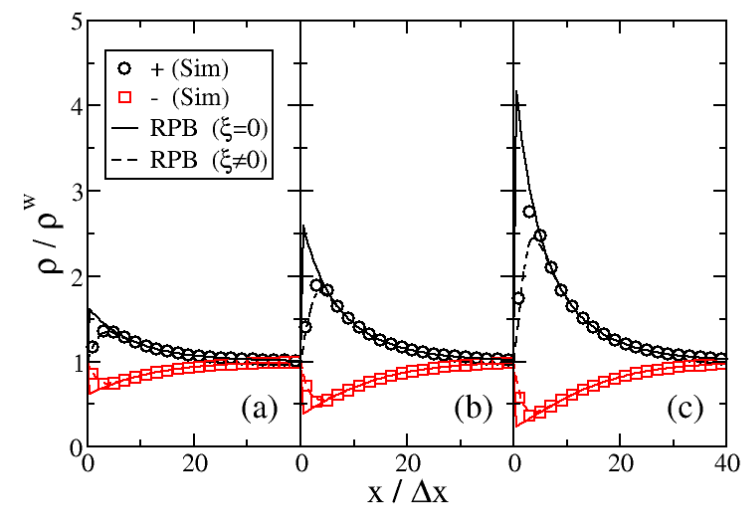

FIG. 7: Ionic profiles in the water phase for $\gamma=0.0$, $\beta \Delta \mu_{a v}=0, l_{B}=0.8 \Delta x, \rho^{w} \Delta x^{3}=4.910^{-4}$ and Donnan potentials $\beta e \psi_{D}=-1(\mathrm{a}),-2(\mathrm{~b})$ and -3 (c). Simulation results (symbols) are compared to the RPB result (see text) for a thin interface (solid line) and for $V_{ \pm}^{\text {solv }}$ corresponding to $\phi^{t h}(x)=-\tanh (x / \xi)$ (dashed line) expected for an ion-free interface.

Increasing $\beta e \psi_{D}$ while keeping $\beta \Delta \mu_{a v}=0$ increases the charge separation and the excess of ions 
at the interface compared to the bulk concentration. Before analyzing quantitatively the influence of $\psi_{D}$ on these quantities, let us examine the effect of the finite interface width on the ionic profiles $\rho_{ \pm}(x)$ and the influence of the solvation free energy on the composition profile $\phi(x)$. Fig. 7 reports the ionic profiles for $\gamma=0.0, \beta \Delta \mu_{a v}=0$ and Donnan potentials $\beta e \psi_{D}=-1,-2$ and -3 . Simulation results are compared to the RPB solution for a thin interface $(\xi=0)$ and for solvation potentials $V_{ \pm}^{\text {solv }}$ corresponding to a composition $\phi^{\text {th }}(x)=-\tanh (x / \xi)$ expected for an ion-free interface, where $\xi=\sqrt{2 K / B}$. The main effect of the finite interface width is to smoothen ionic concentration profiles over a distance $\sim \xi$. Beyond this distance the concentrations are equal to the thin interface result. The profiles are better described by the combination of $\psi^{P B}$ with the smoothed solvation potentials, but the agreement deteriorates as $\left|\psi_{D}\right|$ increases. This is because the composition profile $\phi(x)$ also deviates from $\phi^{t h}(x)$ as $\left|\psi_{D}\right|$ increases : The larger the solvation free energy difference (i.e. the larger $\left.\left|\psi_{D}\right|\right)$, the sharper the interface.

We now analyze how the finite width $\xi$ of the interface affects the overall excess of ions near the interface, quantified by the adsorption :

$$
\begin{aligned}
\Gamma=\int_{-\infty}^{0}\left[\rho_{+}(x)+\rho_{-}(x)-2 \rho^{o}\right] \mathrm{d} x \\
\quad+\int_{0}^{\infty}\left[\rho_{+}(x)+\rho_{-}(x)-2 \rho^{w}\right] \mathrm{d} x .
\end{aligned}
$$

Fig. 8 compares the simulated adsorption to the LPB result :

$$
\Gamma^{L P B}=\frac{\kappa}{8 \pi l_{B}} \frac{\left(\beta e \psi_{D}\right)^{2}}{4}
$$

with $\kappa=\kappa_{o}=\kappa_{w}$ and $l_{B}$ the Bjerrum length common to both phases (since $\gamma=0$ ). This result is obtained by expanding $\rho_{ \pm}(x)$ to second order in $\beta \mu_{ \pm}^{P B}$, for the linear expansion yields $\Gamma=0$. The error bars reported in Fig. 8 correspond to the estimate :

$$
\begin{aligned}
\delta \Gamma=\frac{1}{2}( & \int_{-\Delta x}^{0}\left[\rho_{+}(x)+\rho_{-}(x)-2 \rho^{o}\right] \mathrm{d} x \\
& \left.+\int_{0}^{\Delta x}\left[\rho_{+}(x)+\rho_{-}(x)-2 \rho^{w}\right] \mathrm{d} x\right)
\end{aligned}
$$

with $\Delta x$ the lattice spacing. The agreement between the simulation and LPB results is seen to be very good, except at very large $\psi_{D}$. This performance of the LPB approximation even for $\beta e \psi_{D}>1$ where it fails to predict the ionic profiles can be traced back to a compensation of errors. The RPB result, much closer to the simulated ionic profiles, predicts larger adsorptions than the LPB approximation. But the finite interface width smoothens the ionic profiles and therefore diminishes the value of $\Gamma$. In addition, for the largest $\psi_{D}, \Gamma$ is not small compared to the amount of "bulk" ions $\rho^{o} L_{o}+\rho^{w} L_{w} \sim 0.12 \Delta x^{-2}$ so that the simulated system cannot be considered as an isolated interface in contact with infinite reservoirs.

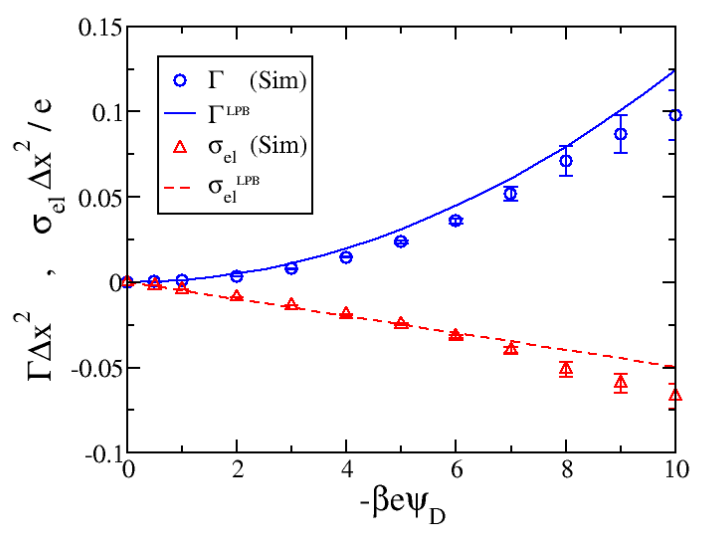

FIG. 8: Ionic adsorption $\Gamma$ and surface charge density $\sigma_{e l}$ of the oil phase as a function of $\psi_{D}$ for $\gamma=0$ and $\beta \Delta \mu_{a v}=0$. Simulation results (symbols) for $l_{B}=0.8 \Delta x$ are compared to the linearized Poisson-Boltzmann results for $\xi=0$ (lines) given by Eqs. (29) and (31).

The good agreement between the simulated $\psi$ and linearized $\mathrm{PB}$ result $\psi^{P B}$ suggests that the electric charge density is also close to the PB solution. In particular, the total electric charge of each phase $\sigma_{e l}=$ $\sigma_{o}=-\sigma_{w}=\int_{-\infty}^{0}\left(\rho_{+}-\rho_{-}\right) e \mathrm{~d} x$ can be compared to the PB prediction for $\xi=0$ :

$$
\frac{\sigma_{e l}^{L P B}}{e}=\frac{\kappa}{4 \pi l_{B} A}\left(\beta e \psi_{D}\right)
$$

with here $A=2$ (see Eq. (27)). Fig. 8 also displays $\sigma_{e l}$ as a function of the Donnan potential $\psi_{D}$ for the simple case $\beta \Delta \mu_{a v}=0$ and $\gamma=0$. The error bars correspond to $\delta \sigma_{e l}=\frac{1}{2} \int_{-\Delta x}^{\Delta x}\left|\rho_{+}-\rho_{-}\right| e \mathrm{~d} x$. This figure shows that simulation results are indeed well described by Eq. (31) and confirms that the charge separation at the interface is proportional to $\psi_{D}$, except at very large $\psi_{D}$. The symmetric interface behaves as a capacitor of permittivity $\epsilon$ and width $\kappa^{-1}$, with capacitance per unit area $\epsilon \kappa$. When submitted to a potential difference $\psi_{D}$, each side builds up a charge per unit area $\sigma_{e l} \propto \epsilon \kappa \psi_{D}$. The good agreement between the simulation and $\mathrm{PB}$ results for $\sigma_{e l}$ is consistent with the finding of ref. [35] that global quantities related to the partitioning between the two phases are not in- 
fluenced by the finite interface width if it is smaller than the interfacial Debye length.

All results presented so far concerned the symmetric solvation case $\beta \Delta \mu_{a v}=0$. Simulations in the asymmetric case also give the expected results. For example, we find that the salt concentration ratio $\rho^{o} / \rho^{w}$ decreases from $e^{-1} \sim 0.37$ for $\beta \Delta \mu_{+}=+2$ and $\beta \Delta \mu_{-}=0$ (hence $\beta \Delta \mu_{a v}=+1$ ) to $e^{-3} \sim 510^{-2}$ for $\beta \Delta \mu_{+}=+4$ and $\beta \Delta \mu_{-}=+2\left(\beta \Delta \mu_{a v}=+3\right)$. While these two conditions correspond to the same $\psi_{D}=-k_{B} T / e$, the former yields a larger adsorption $\Gamma$. In addition, the ionic profiles are again in quantitative agreement with the RPB solution for a shift $s=0$. This choice gives the best agreement when there is no dielectric contrast between the two solvents.

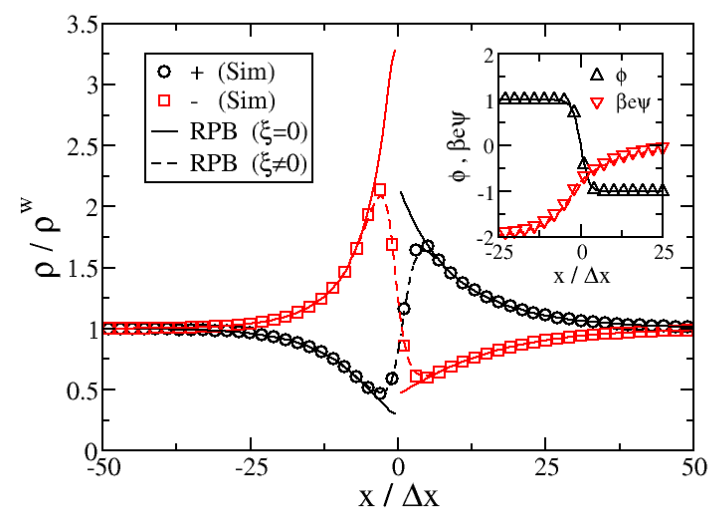

FIG. 9: Ionic profiles at the oil (left) - water (right) interface for $\gamma=0.5, \beta \Delta \mu_{ \pm}= \pm 2, l_{B}=0.8 \Delta x, \rho^{w} \Delta x^{3}=$ $4.910^{-4}$ and a shift $s=-1.25 \Delta x$. Simulation results (symbols) are compared to analytical results for a thin interface (solid line) and for the combination of $\psi^{P B}$ with solvation potentials $V_{ \pm}^{\text {solv }}$ corresponding to a composition $\phi^{t h}(x)=-\tanh (x / \xi)$ (dashed line). The insert compares the simulation (symbols) and analytical (lines) results for the composition $\phi$ and electrostatic potential $\psi$.

When $\gamma \neq 0$ the situation is more complex and even the sign of $s$ depends on $\gamma, \Delta \mu_{+}$and $\Delta \mu_{-}$. An example of such a situation is illustrated in Fig. 9. The best agreement with Eq. (27) was obtained in that case for a shift $s=-1.25 \Delta x$. All other parameters being fixed, we find that for increasing $\Delta \mu_{+}=-\Delta \mu_{-}$ the required shift also increases. Simulation results for the electrostatic potential are well described by the $\mathrm{PB}$ result for a vanishing width $\xi=0$ and a finite shift. Therefore the minimal model of van Roij et al.. to account for image charges seems to be appropriate, at least for the explored range of parameters. Even for $\beta e \psi_{D}>1$, where the fully linear PB approximation fails, we find that the reexponentiated $\mathrm{PB}$ result gives an accurate description of ionic profiles. Moreover, our results show that the finite width of the interface affects the ionic profiles in such a way that the electrostatic potential is still close to the $\mathrm{PB}$ prediction. These profiles are accurately described by the combination of $\psi^{P B}$ with solvation potentials $V_{ \pm}^{s o l v}$ corresponding to an unperturbed composition profile $\phi^{\text {th }}(x)=-\tanh (x / \xi)$. In particular, "far" from the interface, the effect of the finite width on solvation forces is negligible and the ionic profiles coincide with the result for $\xi=0$. As shown previously in the case $\gamma=0$, we expect for larger $\psi_{D}$ a perturbation of the composition profile and a corresponding modification of the ionic concentrations.

The results presented in this section demonstrate the ability of our coarse-grained simulation scheme to study the interface between two immiscible solvents in presence of ions, including the effect of their (possibly asymmetric) affinity for both phases. To assess the validity of the simulation scheme we only showed results in the one-dimensional case, for which analytical predictions are available. Simulation in two or three dimensions is straightforward.

\section{CONCLUSION}

We have presented a coarse-grained simulation method for complex charged systems. This mesoscopic model couples a hydrodynamic description to a free energy functional accounting for the interactions between solvent(s) and charged solutes. All the parameters entering in the model, such as free energy parameters (e.g. related to ion solvation), solvent viscosity or ionic diffusion coefficients are, at least in principle, computable by simulations at the molecular level. We described the implementation of this model in a hybrid lattice-based scheme, whereby the evolution of the overall mass and momentum is taken care of via a Lattice Boltzmann scheme, whereas the composition and ionic concentrations are updated using the linkflux method. We presented several applications of the coarse-grained simulation method: the transport of charged tracers in charged porous media, the deformation of an oil droplet in water under the effect of an applied electric field, and the distribution of ions at an oil-water interface as a function of the ions affinity for both solvents. When possible, we compared our simulation results to exact or approximate analytical results to investigate the range of simulation parameters that can be used to recover the continuous results (e.g. the effect of a finite interface width).

The proposed method will be very useful to sim- 
ulate the dynamics of complex mixtures of solvents and ions. In particular, it will be interesting to investigate electrokinetic phenomena at a charged oilwater interface, or of oil-water mixtures in charged porous media. Understanding electrokinetic effects in these systems might be very helpful in designing new electro-acoustic oil recovery techniques or monitoring devices. This coarse-grained simulation method could also be applied to the study of electrowetting and of microfluidic devices, particularly those based on electrokinetic pumping.

Although surfactant free emulsions can be stabilized by the presence of salt, the most usual situation also involves sufactant molecules at the interface and in solution. As free energy based models for mixtures of oil, water and surfactants have already been proposed, it should be rather straightforward to couple them to the one introduced in the present paper. One physical feature not included in the method presented here is the presence of thermal fluctuations. It would be interesting to investigate the possibility of including fluctuations as is done in the fluctuating latticeBoltzmann method [63, 64].

\section{Acknowledgements}

The authors would like to thank Rene van Roij, Jean-Pierre Hansen and Edo Boek for very useful discussions. B.R. acknowledges financial support from the Agence Nationale pour la Gestion des Déchets Radioactifs (ANDRA, France). I.P. acknowledges financial support from CAICYT (FIS2005-01299). The work of the FOM Institute is part of the research program of FOM and is made possible by financial support from the Netherlands organization for Scientific Research (NWO).

[1] M. Karttunen, I. Vattulainen and A. LakkariNEN, Novel Methods in Soft Matter Simulations, Lecture Notes in Physics Vol. 640, Springer Verlag, 2004.

[2] P. Español and P. Warren, Europhys. Lett. 30, 191 (1995).

[3] C. Lowe, Europhys. Lett. 47, 145 (1999).

[4] A. Malevanets and R. Kapral, J. Chem. Phys. 112, 7260 (2000).

[5] S. SuccI, The Lattice Boltzmann Equation for Fluid Dynamics and Beyond, Oxford University Press, 2001.

[6] M. Swift, E. Orlandini, and J. Yeomans, Phys. Rev. Lett. 75 (1995).

[7] E. Orlandini, M. Swift, and J. Yeomans, Europhys. Lett. 32, 463 (1995).

[8] M. Swift, E. Orlandini, W. Osborn, and J. YeOMAns, Phys. Rev. E 54, 5041 (1996).
[9] O. Theissen, G. Gompper, and D. Kroll, Europhys. Lett. 42, 419 (1998).

[10] A. Lamura, G. Gonnella, and J. Yeomans, Europhys. Lett. 45, 314 (1999).

[11] R. VAN DER SMAN and S. VAN DER GraAf, Rheol. Acta 46, 3 (2006).

[12] C. Denniston, E. Orlandini, and J. M. Yeomans, Phys. Rev. E 63, 056702 (2001).

[13] C. M. Care, I. Halliday, K. Good, and S. V. Lishchuk, Phys. Rev. E 67, 061703 (2003).

[14] A. Dupuis, D. Marenduzzo, E. Orlandini, and J. M. Yeomans, Phys. Rev. Lett. 95, 097801 (2005).

[15] T. J. Spencer and C. M. Care, Phys. Rev. E 74, 061708 (2006).

[16] Q. Li and A. Wagner, Phys. Rev. E 76, 036701 (2007).

[17] S. Ramachandran, P. Kumar, and I. PagonabarRAga, Eur. Phys. J. E 20, 151 (2006).

[18] J.-C. Desplat, I. PAgonabarraga, and P. Bladon, Comp. Phys. Comm. 134, 273 (2001).

[19] J. Harting, M. Venturoli, and P. Coveney, Phil. Trans. Roy. Soc. A 362, 1703 (2004).

[20] K. Stratford, R. Adhikari, I. Pagonabarraga, and J.-C. Desplat, J. Stat. Phys. 121, 163 (2005).

[21] K. Stratford, R. Adhikari, I. Pagonabarraga, J.-C. Desplat, and M. E. Cates, Science 309, 2198 (2005).

[22] K. Stratford and I. Pagonabarraga, Comp. Math. App. 55, 1585 (2008).

[23] J. Horbach and D. Frenkel, Phys. Rev. E 64, 061507 (2001).

[24] C. Pooley and K. Furtado, Phys. Rev. E 77, 046702 (2008).

[25] F. Capuani, I. Pagonabarraga, and D. Frenkel, J. Chem. Phys. 121, 973 (2004).

[26] I. Pagonabarraga, F. Capuani, and D. Frenkel, Comp. Phys. Comm. 169, 192 (2005).

[27] A. Onuki, Phys. Rev. E 73, 021506 (2006).

[28] A. Onuki, J. Chem. Phys. 128, 224704 (2008).

[29] A. Onuki, Europhys. Lett. 82, 58002 (2008).

[30] R. Ledesma-Aguilar, A. Hernández-Machado, and I. Pagonabarraga, Phys. Fluids 19, 102112 (2007).

[31] A. Briant and J. Yeomans, Phys. Rev. E 69, 031603 (2004).

[32] R. van der Sman and S. van Der Graaf, Comput. Phys. Comm. 178, 492 (2008).

[33] N. Deneshyuk and J.-P. Hansen, J. Chem. Phys. 121, 3613 (2004).

[34] J. ZwanikKen and R. van RoiJ, Phys. Rev. Lett. 99, 178301 (2007).

[35] M. Bier, J. Zwanikken, and R. van RoiJ, Phys. Rev. Lett. 101, 046104 (2008).

[36] U. M. B. Marconi and P. Tarazona, J. Chem. Phys. 110, 8032 (1999).

[37] U. Marconi and P. Tarazona, J. Phys.: Condens. Matter 12, A413 (2000).

[38] H. Lowen, J. Phys.: Condens. Matter 15, V1 (2003).

[39] A. J. Archer and R. Evans, J. Chem. Phys. 121, 4246 (2004).

[40] M. Rex and H. Löwen, Physical Review Letters 101, 
148302 (2008).

[41] K. Furtado, C. Pooley, and J. Yeomans, Phys. Rev. E 78, 046308 (2008).

[42] D. Marenduzzo, E. Orlandini, M. E. Cates, and J. M. Yeomans, Phys. Rev. E 76, 031921 (2007).

[43] H. Li and H. KI, Comm. Comput. Phys. 4, 337 (2008).

[44] R. Benzi, S. Succi, and M. Vergassola, Phys. Rep. 222, 145 (1992).

[45] P. Bhatnagar, E. Gross, and M. Krook, Phys. Rev. 94, 511 (1954).

[46] W. H. Press, S. A. Teukolsky, W. T. VetterLING, and B. P. Flannery, Numerical Recipes in C: The Art of Scientific Computing, Cambridge University Press, Cambridge, 2nd edition, 1993.

[47] B. Rotenberg, I. Pagonabarraga, and D. Frenkel, Europhys. Lett. 83, 34004 (2008).

[48] P. Sen, Conc. Magn. Reson. Part A 23A, 1 (2004).

[49] P. Mitra, P. Sen, S. L.M., and P. Le Doussal, Phys. Rev. Lett. 68, 3555 (1992).

[50] M. Ochs, M. Boonekamp, H. Wanner, H. Sato, and M. YuI, Radiochimica Acta 82, 437 (1998).

[51] I. C. Bourg, A. C. M. Bourg, and G. Sposito, J. Cont. Hyd. 61, 293 (2003).

[52] I. Bourg, G. Sposito, and A. Bourg, Clay Clay Miner. 54, 363 (2006).

[53] M. Van der Hoef and D. Frenkel, Phys. Rev. A
41, 4277 (1990).

[54] M. VAn Der Hoef and D. Frenkel, Physica D 47, 191 (1991).

[55] C. Lowe and D. Frenkel, Physica A 220, 251 (1995).

[56] A. Valfouskaya, P. Adler, J.-F. Thovert, and M. Fleury, J. App. Phys. 97, 083510 (2005).

[57] C. O'Konski and H. T. JR., J. Phys. Chem. 57, 955 (1953).

[58] G. Marcus, S. Samin, and Y. Tsori, J. Chem. Phys. 129, 061101 (2008).

[59] G. Luo, S. Malkova, J. Yoon, D. Schultz, B. Lin, M. Meron, I. Benjamin, P. VanÝsek, and M. Schlossman, Science 311, 216 (2006).

[60] M. Leunissen, A. VAn BlaAderen, A. Hollingsworth, M. Sullivan, and P. Chaikin, Proc. Natl. Acad. Sci. USA 104, 2585 (2007).

[61] J. DE GraAf, J. ZWANIKKen, M. Bier, A. BaArsma, Y. Oloumi, M. Spelt, and R. van RoiJ, J. Chem. Phys. 129, 194701 (2008).

[62] J.-P. Hansen and I. McDonald, Theory of Simple Liquids, 3rd Edition, Academic Press, 2006.

[63] R. Adhikari, K. Stratford, M. E. Cates, and A. J. Wagner, Europhys. Lett. 71, 473 (2005).

[64] B. Dünweg, U. Schiller, and A. Ladd, Phys. Rev. E 76, 036704 (2007). 